\title{
Comparing Hippocampal Atrophy in Alzheimer's Dementia and Dementia with Lewy Bodies
}

\author{
Nicole Chow ${ }^{\mathrm{a}, \mathrm{b}}$ Dag Aarsland ${ }^{\mathrm{e}, \mathrm{h}, \mathrm{k}}$ Hedieh Honarpisheh ${ }^{\mathrm{d}}$ Mona K. Beyer $^{\mathrm{i}}$ \\ Johanne H. Somme $\quad$ David Elashoffc Arvid Rongve ${ }^{f} \quad$ Ole B. Tysnes ${ }^{g}$ \\ Paul M. Thompson ${ }^{a, b} \quad$ Liana G. Apostolova ${ }^{a, b}$ \\ aDepartment of Neurology, ${ }^{\mathrm{b}}$ Imaging Genetics Center, Laboratory of Neuroimaging, David Geffen School of \\ Medicine and 'Division of General Internal Medicine and Health Services Research, UCLA, Los Angeles, Calif., \\ and Pathology and Laboratory Medicine, Yale School of Medicine, New Haven, Conn., USA; Institute of Clinical \\ Medicine, University of Oslo, Oslo, fDepartment of Psychiatry, Haugesund Hospital, Haugesund, 9Department

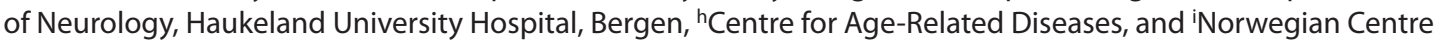 \\ for Movement Disorders, Stavanger University Hospital, Stavanger, Norway; 'Department of Neurology, Cruces \\ University Hospital, Baraclado, Spain; ${ }^{2}$ Department of Neurobiology, Ward and Care Services, Alzheimer's Disease \\ Research Centre, Karolinska Institutet, Stockholm, Sweden
}

\section{Key Words}

Alzheimer's disease - Dementia with Lewy bodies ·

Hippocampus $\cdot$ Magnetic resonance imaging $\cdot$ Atrophy

\begin{abstract}
Background/Aims: Dementia with Lewy bodies (DLB) and Alzheimer's disease (AD) are the two most common neurodegenerative dementias. During the early stages, clinical distinction between them is often challenging. Our objective was to compare hippocampal atrophy patterns in mild $A D$ and mild DLB. We hypothesized that DLB subjects have milder hippocampal atrophy relative to $A D$ subjects. Methods: We analyzed the T1-weighted magnetic resonance imaging data from 113 subjects: 55 AD, 16 DLB and 42 cognitively normal elderly (normal controls, NC). Using the hippocampal radial distance technique and multiple linear regression, we analyzed the effect of clinical diagnosis on hippocampal radial distance, while adjusting for sex and age. Three-dimensional statistical maps were adjusted for multiple comparisons using permutation-based statistics with a threshold of $p<0.01$. Results: Compared to NC, AD
\end{abstract}

exhibited significantly greater atrophy in the cornu ammonis (CA) 1, CA2-3 and subicular regions bilaterally while DLB showed left-predominant atrophy in the $C A 1$ region and subiculum. Compared directly, AD and DLB did not reveal statistically significant differences. Conclusion: Hippocampal atrophy in mildly impaired DLB subjects is less severe than atrophy seen in mildly impaired $A D$ subjects. Both $A D$ and DLB show predominant atrophy of the CA1 subfield and subiculum.

Copyright $\odot 2012$ S. Karger AG, Basel

\section{Introduction}

Dementia with Lewy bodies (DLB) - the second most common neurodegenerative dementia after Alzheimer's disease (AD), has a complex clinical presentation including psychiatric, motor, sleep and autonomic disturbances in addition to cognitive impairment [1]. Prognosis and quality of life in DLB are generally even poorer than in

Research was conducted at: Department of Neurology, David Geffen School of Medicine, UCLA, Los Angeles, Calif., USA

\section{KARGER \\ Fax +41613061234 \\ E-Mail karger@karger.ch}

www.karger.com
(C) 2012 S. Karger AG, Basel

$1420-8008 / 12 / 0341-0044 \$ 38.00 / 0$

Accessible online at:

www.karger.com/dem
Liana G. Apostolova, MD, MSCR

Mary S. Easton Center Alzheimer's for Alzheimer's Disease Research

10911 Weyburn Ave, 2nd floor

Los Angeles CA 90095 (USA)

Tel. +1 310794 2551, E-Mail lapostolova@mednet.ucla.edu 
$\mathrm{AD}$ [2], as are health-related costs [3] and mortality [4]. DLB patients have a different response to drug treatment and are at a particularly high risk of developing severe hypersensitivity reactions to neuroleptic drugs [5].

Differentiating DLB from other dementias, including $\mathrm{AD}$, is of particular clinical importance. However, this distinction can be difficult, especially early in the disease course when the classic DLB clinical profile may not yet be fully developed [6]. Consequently, many pathologically confirmed cases of DLB have been clinically misdiagnosed as $\mathrm{AD}$ premortem [7-10]. While the clinical consensus criteria for DLB were recently revised [1] and preliminary evidence suggests that the newly revised criteria have greater sensitivity [11], a systematic evaluation of their sensitivity and specificity is not yet available.

The ability of a range of biomarkers to aid in the differential diagnosis of DLB is actively being explored. The most established method to date, dopamine transporter single-photon-emission computerized tomography [12], is relatively expensive and is not readily available at all centers. Structural neuroimaging using magnetic resonance imaging (MRI) is among the most established biomarkers for $\mathrm{AD}$ and is now used for the diagnosis of prodromal AD [13]. Novel sensitive MRI analytic techniques have been recently developed and have enabled us to identify presymptomatic and early symptomatic structural changes in $\mathrm{AD}$ [14-16].

Few studies have compared the MRI changes in DLB and $\mathrm{AD}$; most have used either visual rating, the regionof-interest approach, or whole brain imaging analysis methods such as voxel-based morphometry or cortical thickness approaches to measure cortical or subcortical atrophy in DLB compared to normal controls (NC) or $\mathrm{AD}$. These types of studies find that relative to $\mathrm{AD}, \mathrm{DLB}$ subjects showed significantly less atrophy in the orbitofrontal and temporal lobes [17-19]. In addition, temporal lobe and hippocampal atrophy are less pronounced in DLB than in AD [19-22], but the magnitude of the differences is small, precluding the ability of standard volumetric hippocampal assessments to readily distinguish between AD and DLB. Yet one recent study suggested that visual scoring of medial temporal atrophy on MRI obtained approximately 1.5 years prior to death might provide discriminatory power for distinguishing patients with moderately severe pathologically confirmed AD (Mini-Mental State Examination, MMSE 13.8 $\pm 4.54)$ from those with moderately severe pathologically confirmed DLB (MMSE $13.3 \pm 7.83$ ) and mild vascular cognitive impairment (MMSE $22.8 \pm 4.36$ ) [21]. Another study applied a recently developed hippocampal partial subfield segmentation technique to the 3-tesla MRI data of 16 AD (mean MMSE 21.5, range 16-27) and 16 DLB (mean MMSE 18, range 15-27) patients and 16 NC subjects (mean MMSE 29, range 26-30) [23]. Their measurements were limited to the three most anterior contiguous coronal slices from the hippocampal body. Despite significant technical difficulties in ascertaining the CA1 subfield boundary, the authors reported significantly smaller CA1 area in AD when compared to DLB.

Most prior MRI studies comparing DLB and $\mathrm{AD}$ have included patients with moderately severe dementia. However, recent advances in biomarker development and structural neuroimaging, in particular, now allow us the unprecedented opportunity to detect structural changes in the presymptomatic stages for other dementing disorders such as $\mathrm{AD}[15,24]$. In this study, we analyzed the imaging data of DLB and AD subjects in the mild dementia stages. Our objective was to compare hippocampal atrophy patterns in mild AD and mild DLB. We hypothesized that DLB subjects would have milder hippocampal atrophy relative to subjects diagnosed with $\mathrm{AD}$, with potentially greater involvement of the CA2-3 subfields, as was recently suggested by one postmortem study [21].

\section{Methods}

Subjects

The Dementia study in western Norway (DemWest) is a multisite longitudinal study of the natural history and biological correlates of dementia. Details of inclusion and assessment procedures have been published previously [25]. In brief, 196 subjects with mild dementia - defined as an MMSE [26] score of 20 or higher - were recruited from referrals to all geriatricmedicine, old-age-psychiatry and neurology outpatient clinics in Rogaland and Hordaland counties in western Norway between March 2005 and March 2007. The study was later enriched with more DLB subjects, including some in the moderate dementia stages (MMSE range 18-20). Standardized clinical instruments were employed to detect and rate cognition, psychiatric and motor symptoms as previously described [25]. Diagnosis of AD was made according to the National Institute of Neurological and Communication Disorders and Stroke and the $\mathrm{AD}$ and Related Disorders Association (NINCDSADRDA) criteria [27] and diagnosis of DLB according to the revised DLB consensus criteria [1]. Two research psychiatrists independently applied the diagnostic criteria twice - at baseline and after 1 year. In cases of disagreement, and whenever more than one set of operationalized diagnostic criteria was met, final diagnostic ascertainment was made based on consensus between the 2 physicians after careful review of all available information. All DemWest patients were actively recruited 
to autopsy. To date, 7 patients have undergone postmortem exams using standard methods and diagnostic criteria, as previously described $[28,29]$. In all 7 cases, the pathological diagnosis agreed with the premortem clinical diagnosis. Our study analyzed the imaging data of $55 \mathrm{AD}$ and 16 DLB DemWest subjects who provided a baseline structural MRI scan of sufficient quality for imaging analyses.

Our control group consisted of 42 cognitively normal elderly subjects who were enrolled in the ParkWest study - another longitudinal project in western Norway. These NC subjects were scanned during the same time period on the same scanners and with the same imaging protocol as our DemWest subjects. ParkWest NC were free from parkinsonism, dementia, major depression and psychosis and scored within the cognitively normal range on the detailed ParkWest neuropsychological battery which has been previously described [30]. Both studies were approved by the regional Norwegian committee for medical research ethics for western Norway. All subjects gave written informed consent for the participation in the study after procedures had been explained in detail in accordance with the Declaration of Helsinki.

Imaging Data Collection and Analysis

Subjects were scanned at 5 different sites located in Stavanger, Haugesund, Haraldsplass, Bergen and Arendal. The following protocols were used:

- Stavanger: 1.5 T Philips Intera (Best, The Netherlands), repetition time (TR)/echo time (TE) 10.0/4.6 ms, flip angle $30^{\circ}, 2-\mathrm{mm}$ slices, $1-\mathrm{mm}$ gap, number of excitations (NEX) 2 , matrix $256 \times 256$, nominal resolution is $1 \times 1 \times 1.28 \mathrm{~mm}$.

- Haugesund: $1.5 \mathrm{~T}$ Philips Intera (Best, The Netherlands), TR/TE 20.0/4.6 ms, flip angle $30^{\circ}$, 1-mm slice thickness with no gap, NEX 1, matrix $256 \times 256$, nominal resolution is $1 \times 1 \times 1.46 \mathrm{~mm}$.

- Haraldsplass: $1.5 \mathrm{~T}$ General Electric Signa Excite (Milwaukee, Wisc., USA), TR/TE $8.2 / 3.1 \mathrm{~ms}$, flip angle $7^{\circ}$, 1 -mm slice thickness, 1 -mm gap, NEX 1, matrix $256 \times 256$, nominal resolution is $1 \times 1 \times 1.29 \mathrm{~mm}$.

- Bergen: 1.5 T General Electric Signa Excite (Erlangen, Germany), TR/TE 8.2/3.1 ms, flip angle 7 degrees, $1-\mathrm{mm}$ slice thickness with no gap, NEX 1, matrix $256 \times 256$, nominal resolution is $1 \times 1 \times 1.33 \mathrm{~mm}$.

- Arendal: 1.0 T Philips Intera (Best, The Netherlands), TR/ TE $25 / 6.9 \mathrm{~ms}$, flip angle $30^{\circ}, 2-\mathrm{mm}$ slice thickness with no gap, NEX 1, matrix $256 \times 256$, nominal resolution is $1 \times 1 \times$ $1.28 \mathrm{~mm}$.

T2-weighted and fluid-attenuated inversed recovery (FLAIR) sequences were collected to evaluate subjects for strokes and/or structural lesions. Subjects with these findings were excluded from our imaging analyses, as were those with baseline scan artifacts or scans of insufficient quality. Our final cohort of DemWest subjects consisted of 71 patients. There were no significant differences in age, sex, education, disease duration and MMSE scores between DemWest subjects who underwent an MRI and those who did not.

Individual MRI scans were automatically registered to the International Consortium for Brain Mapping 53 (ICBM53) template, an average of 53 normal adult brains, using a 9-pa- rameter transformation. This step orients each brain volume into the ICBM53 standardized coordinate system by rotating and globally scaling to correct for differences in head tilt and head size between subjects. Next, hippocampi were manually traced on coronal slices by one researcher $\mathrm{HH}$, intrarater reliability Cronbach's alpha $=0.9$ ) blinded to the age, sex, education, MMSE score and diagnosis of subjects, and following our detailed hippocampal tracing protocol as previously described [31]. The traces included the hippocampus proper, dentate gyrus and subiculum. Traces were converted into hippocampal contours and transformed into 3-dimensional parametric surface mesh models, which were then separated into top and bottom components [32]. These mesh models assured normalization of the spatial frequency of the digitized surface points. Next, a medial core, threading down the center of the hippocampus, was computed. Radial distance was measured from the medial core to the surface of the hippocampus. Each radial distance value was recorded at the corresponding surface coordinate point. These resulting individual hippocampal radial distance maps were combined across subjects to create group average distance maps for quantitative comparisons of surface morphology between diagnostic groups [32].

\section{Intra- and Inter-Scanner Reliability Analyses}

Human phantom scanning of 3 cognitively normal individuals was performed to test scanner reliability. Each volunteer was scanned twice with all scanners included in this study. We performed interscanner reliability analyses. The hippocampi were manually traced (JHS, interrater reliability Cronbach's alpha $=0.896)$ and the volumes obtained as previously described. Intersite Cronbach's alpha for hippocampal volumes was 0.967 .

\section{Statistical Methods}

One-way analyses of variance (ANOVA) with post hoc Bonferroni correction for multiple comparisons were run to examine between-group diagnostic differences in continuous variables such as age, education and MMSE scores. A $\chi^{2}$ test was used to determine differences in sex distribution. The effect of diagnosis on hippocampal radial distance was studied by means of linear regression while correcting for demographic variables that showed significant between-group differences. Our 3-dimensional statistical maps were adjusted for multiple comparisons, using permutation-based statistics with a threshold of $\mathrm{p}<0.01$.

\section{Results}

Mean demographic data for the diagnostic groups NC $(\mathrm{n}=42), \mathrm{AD}(\mathrm{n}=55)$ and DLB $(\mathrm{n}=16)$ are shown in table 1 . The AD and DLB subjects were well matched for overall cognitive impairment as assessed by MMSE (AD $23.9 \pm 2.2$, DLB $23 \pm 2.7, \mathrm{p}=0.4$ ), the Clinical Dementia Rating (CDR) global score (AD $0.76 \pm 0.30$, DLB 0.80 $\pm 0.25, \mathrm{p}=0.671)$ and CDR Sum of Boxes (AD $4.39 \pm$ 
Table 1. Demographic characteristics for the diagnostic comparisons study

\begin{tabular}{lcccc}
\hline Variable & $\mathrm{NC}(\mathrm{n}=42)$ & $\mathrm{AD}(\mathrm{n}=55)$ & $\mathrm{DLB}(\mathrm{n}=16)$ & $\mathrm{p}$ value \\
\hline Age, years & $69.86 \pm 6.756(50-82)$ & $74.76 \pm 7.371(55-89)$ & $78.13 \pm 6.479(69-88)$ & $<0.0001$ \\
Sex, M:F & $14: 28$ & $13: 42$ & $9: 7$ & 0.046 \\
Education, years & $10.214 \pm 2.526(7-17)$ & $9.482 \pm 2.453(7-18)$ & $8.531 \pm 1.803(7-12)$ & 0.053 \\
MMSE $^{\mathrm{a}}$ & $28.81 \pm 1.110(27-30)$ & $23.85 \pm 2.155(19-29)$ & $23.00 \pm 2.683(18-26)$ & $<0.0001(\mathrm{AD}$ vs. DLB, $\mathrm{p}=0.4)$ \\
CDR $^{\mathrm{b}}$ & $\mathrm{N} / \mathrm{A}$ & $0.76 \pm 0.302(1-2)$ & $0.80 \pm 0.254(1-1)$ & 0.671 \\
CDR Sum of Boxes $^{\mathrm{b}}$ & $\mathrm{N} / \mathrm{A}$ & $4.39 \pm 1.931(2-9)$ & $5.03 \pm 1.986(3-10)$ & 0.260 \\
\hline
\end{tabular}

a Two subjects (one AD and one DLB) had MMSE = 19 and one DLB subject had MMSE = 18 .

${ }^{\mathrm{b}}$ CDR and CDR Sum of Boxes available for 15 DLB subjects.

1.93, DLB $5.03 \pm 1.99, \mathrm{p}=0.260)$. Significant differences in age were found, with the DLB group being the oldest and the NC group being the youngest ( $\mathrm{p}<0.0001$ ). Sex distribution was also significantly different with the DLB group having more men than women, while the opposite was true for the AD and NC groups $(\mathrm{p}=0.026)$. As expected, NC subjects had a significantly higher mean MMSE score $(28.81 \pm 1.1)$ than AD $(23.9 \pm 2.2)$ and DLB subjects $(23.0 \pm 2.7)(\mathrm{p}<0.0001)$, but there was no significant difference between $\mathrm{AD}$ and DLB. Age and sex were included as covariates in our radial distance multiple regression models.

Compared to NC, the AD group exhibited significantly greater atrophy in the CA1, CA2-3 and subicular region bilaterally (left $\mathrm{p}_{\text {corrected }}<0.0001$, right $\mathrm{p}_{\text {corrected }}=$ 0.0001; fig. 1, 2nd row), while the DLB group showed greater left than right atrophy localizing mainly to the CA1 region and subiculum (left $\mathrm{p}_{\text {corrected }}=0.0004$, right $\mathrm{p}_{\text {corrected }}=0.056$; fig. 1, 3rd row). Quantitatively, both DLB and AD groups had between $10-40 \%$ smaller radial distance in the statistically significant areas relative to NC. The AD versus DLB comparison did not reveal statistically significant between-group differences (fig. 1, bottom row). The average and standard deviation radial distance maps for each group can be seen in figure 2 .

\section{Discussion}

Differentiating DLB from $\mathrm{AD}$ early in the disease course can be challenging, as the complete clinical profile of DLB may not yet be fully developed. Here, we used an advanced surface-based technique to determine if characteristic structural changes are present in the mild dementia stages of DLB and AD that might aid clinicians in their differential diagnosis. We observed significant hippocam- pal atrophy in both diagnostic groups compared to NC. Our mild AD subjects showed greater atrophy in all hippocampal subfields as previously demonstrated [33]. Our mild DLB subjects showed significant atrophy of the CA1 and subiculum relative to NC. Another study, by Sabattoli et al. [22], using the same hippocampal radial distance technique, investigated the atrophy pattern in 14 mild-tomoderate DLB subjects (MMSE range 13-29) relative to $\mathrm{NC}$ and reported atrophy restricted to the anterior portions of CA1. Despite enrolling a cognitively milder DLB cohort (MMSE range 18-26), our study was able to detect more widespread atrophy of the CA1 and subiculum.

Our DLB and AD groups were well matched for overall cognitive impairment, allowing a fair diagnostic comparison. However, with the sample size of the DLB group $(\mathrm{n}=16)$, we failed to show statistically significant differences between the groups. To further analyze the failure to detect significant differences between $\mathrm{AD}$ and $\mathrm{DLB}$, we constructed a confidence interval (CI) of hippocampal volumes to determine the range of likely values for which we would expect to find a difference between the groups. The difference between the left volumes of $\mathrm{AD}$ and DLB had a $95 \%$ CI of $59.0 \pm 340.2 \mathrm{~mm}^{3}$ and the right volume difference had a $95 \%$ CI of $-69.2 \pm 349.4 \mathrm{~mm}^{3}$. These intervals indicate that, at most, the difference between groups was $10 \%$. Using the average volume for each subject, the CI for the difference in means is $-5.1 \pm$ $315 \mathrm{~mm}^{3}$. With this interval, the difference between the groups was calculated to be $7.5 \%$, which is in agreement with the percent $A D$ vs. DLB difference map seen in the last row of Figure 1. Therefore we can conclude that in our study, the AD and DLB groups are indeed very similar in terms of hippocampal volume. The calculated CIs exclude the possibility that these groups are substantially different from one another. While these findings seem to disagree with those reported in other studies [23], there 


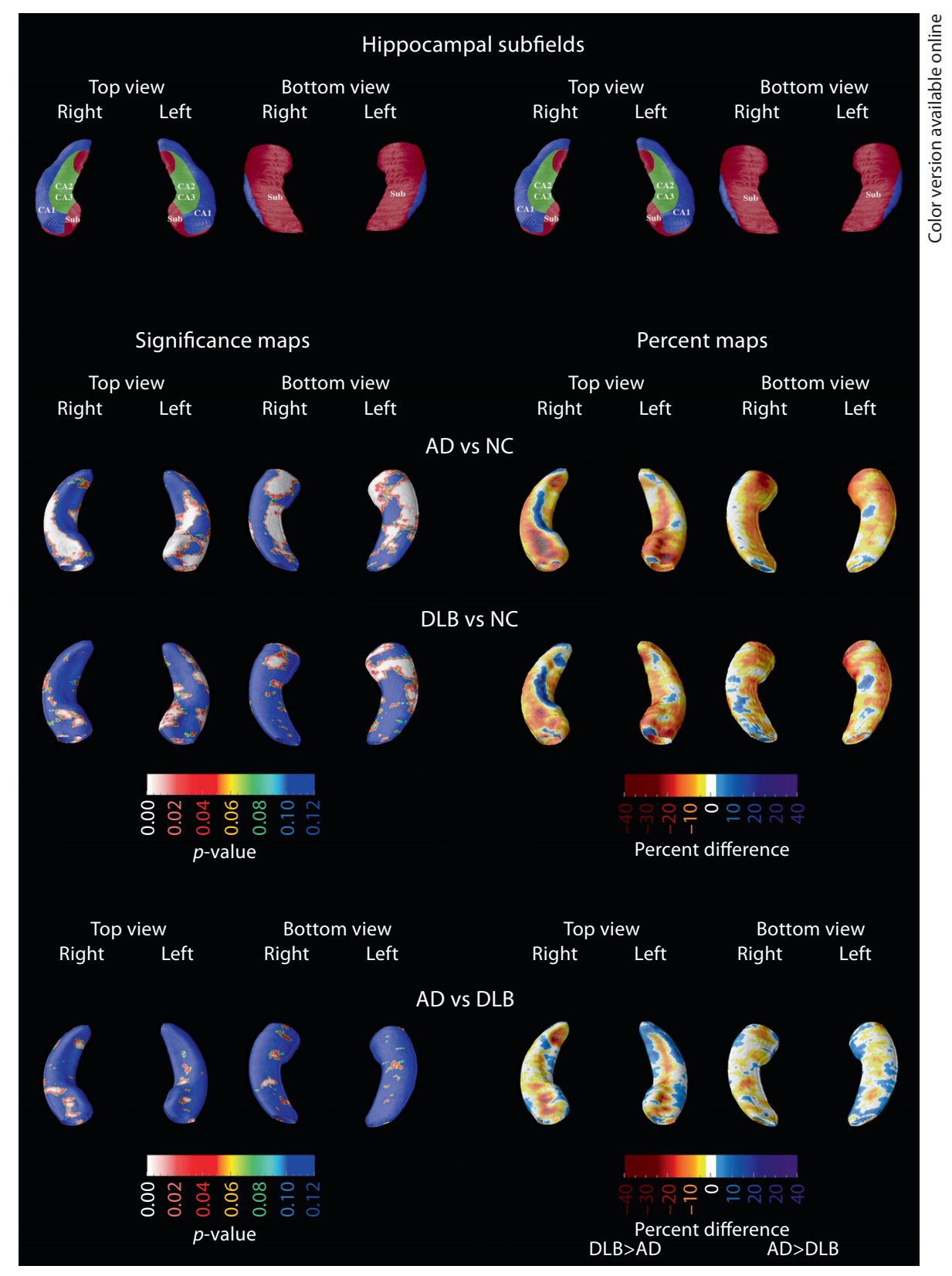

Fig. 1. Three-dimensional statistical and percent difference maps. Red and white areas in the significance maps show statistical significance $(\mathrm{p}<0.05)$. A schematic representation of the hippocampal subfields mapped onto the hippocampal surface is presented in the top row (CA1 in blue, CA2 and CA 3 in green, subiculum in red) [definitions based on 38-40].

are several plausible explanations for this. Firstly, DLB is a heterogeneous disorder with the majority of cases showing, in addition to Lewy bodies, pathological characteristics of $\mathrm{AD}$ such as amyloid plaques and neurofibrillary tangles. Mixed DLB/AD cases frequently show hippocampal atrophy and neurofibrillary tangle pathology of a severity similar to that seen in AD [21]. As such, studies that have relatively small sample sizes could have a study population skewed towards either pure or mixed DLB. Neither our study nor the study by Firbank et al.
[23] had postmortem diagnostic verification to ascertain the pathological diagnoses of our subjects. The study by Firbank et al. [23] used a recently developed method for subfield tracing that involves CA1, CA2 and CA3/4 subfield differentiation on only 3 contiguous coronal sections from the hippocampal structure, with tracing beginning on the first slice where the hippocampal head is no longer visible. Such sparse sampling of the hippocampal structure potentially driven by the tediousness and substantial technical difficulties behind subfield 


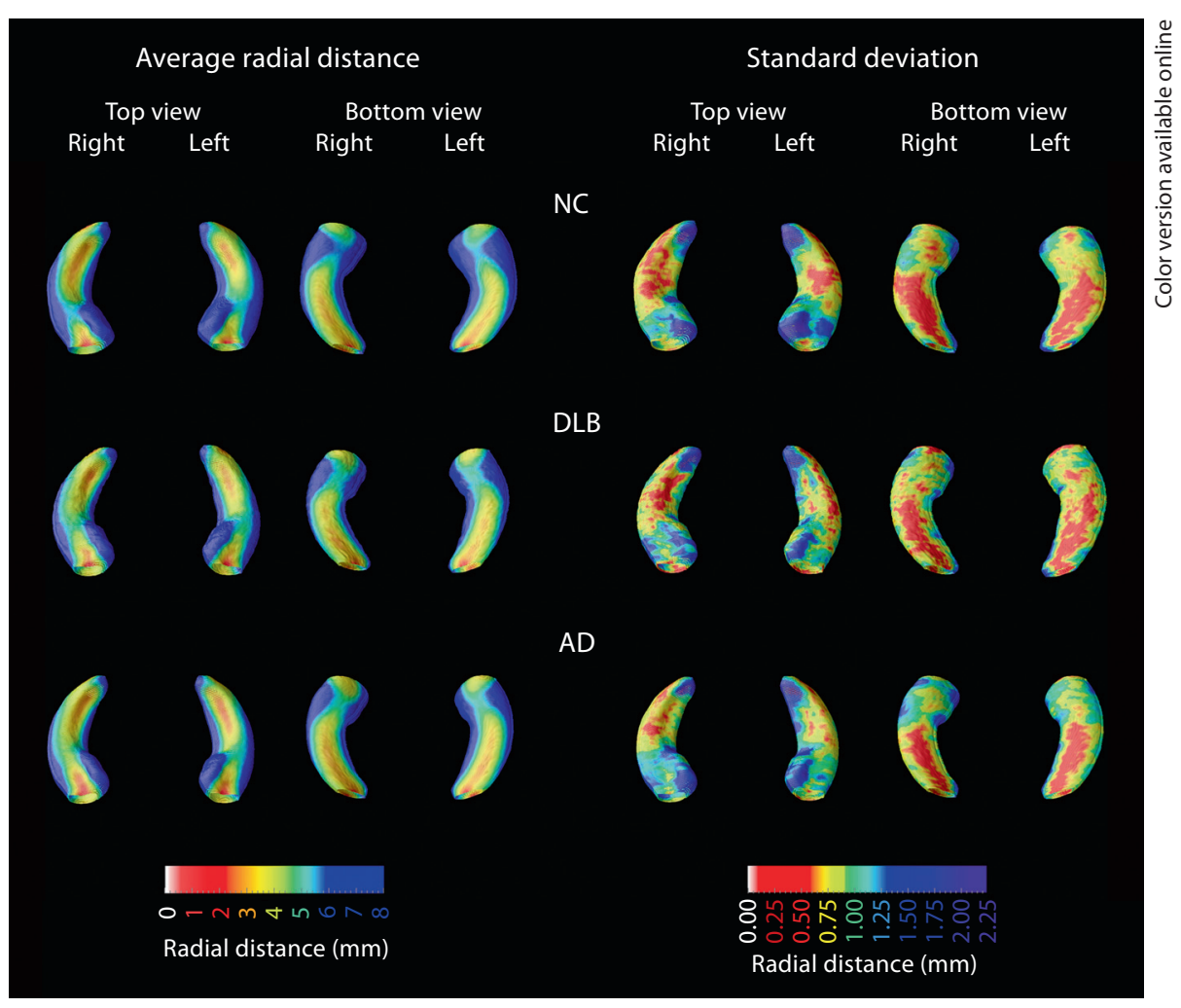

Fig. 2. Average radial distance maps and standard deviation maps of each diagnostic group.

agnostic confirmation was completed on the first 7 cases

tracing, even at 4 -tesla $[23,34,35]$, may or may not generalize well to the whole subfield or to the whole hippocampus. Upon close inspection of the $\mathrm{AD}$ versus $\mathrm{DLB}$ comparison (fig. 1, bottom row), one can appreciate that there are significant between-group differences in the superolateral CA1 area adjacent to the right hippocampal head. This is likely very similar to the area where Firbank et al. [23] reported greater atrophy in AD versus DLB. As in $\mathrm{AD}$ [37], neurofibrillary tangles have been linked with hippocampal atrophy in DLB [21]. Yet, postmortem subjects with DLB show only mild neuronal loss in CA1 and minimal neurofibrillary tangle and neurite pathology in the $\mathrm{CA} 2-3$ regions [36].

Several strengths and limitations of our study should be recognized. Major strengths of the study include its design, the well-characterized patient cohort using standardized instruments to validate core and suggestive DLB features, and its focus on the mild dementia stages. The state-of-the-art imaging analysis is another strength; it allows the identification of focal, regionally specific, disease-associated differences. One limitation of our study is the lack of pathological validation for all cases. However, DemWest subjects are routinely approached for postmortem diagnostic assessment. Postmortem dithat came to autopsy, and in all cases, the clinical and pathological diagnosis were in agreement. Finally, we should also recognize that despite our best efforts to control for between-group variability by entering age and sex as potential confounders in the linear regression analyses, some residual variance due to these and other demographic imbalances might still be present.

\section{Acknowledgment}

We would like to acknowledge the Research Council of Norway (grant number 177966), the Western Norway Regional Health Authority (grant number 911218), the Norwegian Parkinson's Disease Association and research grants from Stavanger University Hospital and the Western Norway Regional Health Authority (grant number 911464) for financial support in the design and conducting of the study as well as the collection and management of obtained data. Our work is also supported by NIH (EB008281, AG020098, RC2 AG036535) and NIA P50 A16570, which provided funding for the analysis and interpretation of data. All sources of support were involved in the preparation, review and approval of the manuscript. Finally, we would like to acknowledge Ketil Oppedal and Turi Olene Dalaker for their contribution to the human phantom scans. 


\section{References}

1 McKeith IG, Dickson DW, Lowe J, et al: Diagnosis and management of dementia with Lewy bodies: third report of the DLB Consortium. Neurology 2005;65:1863-1872.

- 2 Bostrom F, Jonsson L, Minthon L, Londos E: Patients with dementia with Lewy bodies have more impaired quality of life than patients with Alzheimer disease. Alzheimer Dis Assoc Disord 2007;21:150-154.

>3 Bostrom F, Jonsson L, Minthon L, Londos E: Patients with Lewy body dementia use more resources than those with Alzheimer's disease. Int J Geriatr Psychiatry 2007;22: 713-719.

-4 Williams MM, Xiong C, Morris JC, Galvin JE: Survival and mortality differences between dementia with Lewy bodies vs Alzheimer disease. Neurology 2006;67:1935-1941.

5 AarslandD, PerryR,LarsenJP, etal:Neuroleptic sensitivity in Parkinson's disease and parkinsonian dementias. J Clin Psychiatry 2005; 66:633-637.

6 Tiraboschi P, Salmon DP, Hansen LA, Hofstetter RC, Thal LJ, Corey-Bloom J: What best differentiates Lewy body from Alzheimer's disease in early-stage dementia? Brain 2006; 129:729-735.

7 Merdes AR, Hansen LA, Jeste DV, et al: Influence of Alzheimer pathology on clinical diagnostic accuracy in dementia with Lewy bodies. Neurology 2003;60:1586-1590.

-8 HohlU, TiraboschiP, Hansen LA, ThalLJ, Corey-Bloom J: Diagnostic accuracy of dementia with Lewy bodies. Arch Neurol 2000;57: 347-351.

9 Lopez OL, Becker JT, Kaufer DI, et al: Research evaluation and prospective diagnosis of dementia with Lewy bodies. Arch Neurol 2002;59:43-46.

$\checkmark 10$ Nelson PT, Jicha GA, Kryscio RJ, et al: Low sensitivity in clinical diagnoses of dementia with Lewy bodies. J Neurol 2010;257: 359-366.

11 Rongve A, Bronnick K, Ballard C, Aarsland $\mathrm{D}$ : Core and suggestive symptoms of dementia with Lewy bodies cluster in persons with mild dementia. Dement Geriatr Cogn Disord 2010;29:317-324.

$\checkmark 12$ Aarsland D, Kurz M, Beyer M, Bronnick K, Piepenstock Nore S, Ballard C: Early discriminatory diagnosis of dementia with Lewy bodies. The emerging role of CSF and imaging biomarkers. Dement Geriatr Cogn Disord 2008;25:195-205.

13 Dubois B, Feldman HH, Jacova C, et al: Research criteria for the diagnosis of Alzheimer's disease: revising the NINCDS-ADRDA criteria. Lancet Neurol 2007;6:734-746.

- 14 Apostolova LG, Dutton RA, Dinov ID, et al: Conversion of mild cognitive impairment to Alzheimer disease predicted by hippocampal atrophy maps. Arch Neurol 2006;63: 693-699.
15 Apostolova LG, Mosconi L, Thompson PM, et al: Subregional hippocampal atrophy predicts Alzheimer's dementia in the cognitively normal. Neurobiol Aging 2010;31: 1077-1088.

16 Apostolova LG, Thompson PM, Green AE, et al: 3D comparison of low, intermediate, and advanced hippocampal atrophy in MCI. Hum Brain Mapp 2010;31:786-797.

17 Ballmaier M, O’Brien JT, Burton EJ, et al: Comparing gray matter loss profiles between dementia with Lewy bodies and Alzheimer's disease using cortical pattern matching: diagnosis and gender effects. Neuroimage 2004;23:325-335.

18 Beyer MK,LarsenJP, AarslandD: Graymatter atrophy in Parkinson disease with dementia and dementia with Lewy bodies. Neurology 2007;69:747-754.

19 Whitwell JL, Weigand SD, Shiung MM, et al: Focal atrophy in dementia with Lewy bodies on MRI: a distinct pattern from Alzheimer's disease. Brain 2007;130:708-719.

20 Barber R, McKeith IG, Ballard C, Gholkar A, O'Brien JT: A comparison of medial and lateral temporal lobe atrophy in dementia with Lewy bodies and Alzheimer's disease: magnetic resonance imaging volumetric study. Dement Geriatr Cogn Disord 2001;12: 198-205.

-21 Burton EJ, Barber R, Mukaetova-Ladinska $\mathrm{EB}$, et al: Medial temporal lobe atrophy on MRI differentiates Alzheimer's disease from dementia with Lewy bodies and vascular cognitive impairment: a prospective study with pathological verification of diagnosis. Brain 2009;132:195-203.

22 Sabattoli F, Boccardi M, Galluzzi S, Treves A, Thompson PM, Frisoni GB. Hippocampal shape differences in dementia with Lewy bodies. Neuroimage 2008;41:699-705.

-23 Firbank MJ, Blamire AM, Teodorczuk A, et al: High resolution imaging of the medial temporal lobe in Alzheimer's disease and dementia with Lewy bodies. J Alzheimers Dis 2010;21:1129-1140.

-24 Apostolova LG, Thompson PM: Mapping progressive brain structural changes in early Alzheimer's disease and mild cognitive impairment. Neuropsychologia 2008;46: 1597-1612.

25 Aarsland D, Rongve A, Nore SP, et al: Frequency and case identification of dementia with Lewy bodies using the revised consensus criteria. Dement Geriatr Cogn Disord 2008; 26:445-452.

26 Folstein M, Folstein S, McHugh PR: Clinical predictors of improvement after electroconvulsive therapy of patients with schizophrenia, neurotic reactions, and affective disorders. Biol Psychiatry 1973;7:147-152.
27 McKhann G, Drachman D, Folstein M, Katzman R, Price D, Stadlan EM: Clinical diagnosis of Alzheimer's disease: report of the NINCDS-ADRDA Work Group under the auspices of the Department of Health and Human Services Task Force on Alzheimer's Disease. Neurology 1984;34:939-944.

28 Kirvell SL, Elliott MS, Kalaria RN, Hortobagyi T, Ballard CG, Francis PT: Vesicular glutamate transporter and cognition in stroke: a case-control autopsy study. Neurology 2010; 75:1803-1809.

29 Hortobagyi T, Troakes C, Nishimura AL, et al: Optineurin inclusions occur in a minority of TDP-43 positive ALS and FTLD-TDP cases and are rarely observed in other neurodegenerative disorders. Acta Neuropathol 2011;121:519-527.

30 Aarsland D, Bronnick K, Larsen JP, Tysnes $\mathrm{OB}$, Alves G: Cognitive impairment in incident, untreated Parkinson disease: the Norwegian ParkWest study. Neurology 2009;72: 1121-1126.

31 Narr KL, van Erp TG, Cannon TD, et al: A twin study of genetic contributions to hippocampal morphology in schizophrenia. Neurobiol Dis 2002;11:83-95.

-32 Thompson PM, Hayashi KM, De Zubicaray GI, et al: Mapping hippocampal and ventricular change in Alzheimer disease. Neuroimage 2004;22:1754-1766.

33 Apostolova LG, Dinov ID, Dutton RA, et al: 3D comparison of hippocampal atrophy in amnestic mild cognitive impairment and $\mathrm{Al}-$ zheimer's disease. Brain 2006;129:2867-2873.

34 Mueller SG, Stables L, Du AT, et al: Measurement of hippocampal subfields and age-related changes with high resolution MRI at $4 \mathrm{~T}$. Neurobiol Aging 2007;28:719-726.

- 35 Mueller SG, Schuff N, Raptentsetsang S, Elman J, Weiner MW: Selective effect of Apo e 4 on CA 3 and dentate in normal aging and Alzheimer's disease using high resolution MRI at 4 T. Neuroimage 2008;42:42-48.

- 36 Lippa CF, Pulaski-Salo D, Dickson DW, Smith TW: Alzheimer's disease, Lewy body disease and aging: a comparative study of the perforant pathway. J Neurol Sci 1997;147: 161-166.

37 Zarow C, Vinters HV, Ellis WG, et al: Correlates of hippocampal neuron number in Alzheimer's disease and ischemic vascular dementia. Ann Neurol 2005;57:896-903.

38 Mai JK, Assheuer J, Paxinos G: Atlas of the Human Brain, ed 2. Amsterdam, Elsevier Academic Press, 2004.

39 Duvernoy HM: The Human Hippocampus: An Atlas of Applied Anatomy. München: JF Bergmann, 1988.

40 West MJ, Gundersen HJ: Unbiased stereological estimation of the number of neurons in the human hippocampus. J Comp Neurol 1990;296:1-22. 